\title{
A NOTE ON BERNSTEIN POLYNOMIAL TYPE APPROXIMATIONS
}

\author{
D. H. TUCKER
}

The Bernstein polynomial of order $n$ for a function $f$ defined on $[0,1]$ is defined by

$$
B_{n}(f(t))=\sum_{m=0}^{n}\left(\begin{array}{c}
n \\
m
\end{array}\right) t^{m}(1-t)^{n-m} f\left(\frac{m}{n}\right)=\sum_{m=0}^{n}\left(\begin{array}{c}
n \\
m
\end{array}\right)\left[\Delta^{n-m} t^{m}\right] f\left(\frac{m}{n}\right)
$$

where

$$
\Delta^{n-m} t^{m}=\sum_{\nu=0}^{n-m}\left(\begin{array}{c}
n-m \\
\nu
\end{array}\right)(-1)^{n-m-v t^{n-\nu}} .
$$

These polynomials provide a method for the uniform approximation of a function $f$ which is continuous on the interval $[0,1]$ and has values in a Banach space $X$ by use of the function sequence $\left\{t^{i}\right\}_{i=0}^{\infty}$. Given a sequence $\Phi=\left\{\phi_{i}\right\}_{i=0}^{\infty}$ of continuous functions from $[0,1]$ in to $B[X, Y]$, the space of bounded linear transformations from a Banach space $X$ in to a Banach space $Y$, we define

$$
B_{n}(\Phi, f)=\sum_{m=0}^{n}\left(\begin{array}{c}
n \\
m
\end{array}\right)\left[\Delta^{n-m} \phi_{m}\right] f\left(\frac{m}{n}\right)
$$

to be the $\Phi$-Bernstein approximation of $f$ of order $n$. In this note we shall consider the question of uniform convergence of such approximations.

Definition. The sequence $\Phi$ is said to satisfy condition A if there exists an $M>0$ such that if $\left\{x_{m}\right\}_{m=0}^{\infty}$ is a bounded sequence in $X$, then

$$
\left\|\sum_{m=0}^{n}\left(\begin{array}{c}
n \\
m
\end{array}\right)\left[\Delta^{n-m} \phi_{m}\right] \cdot x_{m}\right\|_{C(Y)} \leqq M \sup _{(m)}\left\|x_{m}\right\| \quad \text { for all } n \geqq 0 ;
$$

where \|\|$_{C(Y)}$ denotes the uniform norm on the function space $C(Y)$ of continuous functions from $[0,1]$ in to $Y$.

THEOREM. The following two statements are equivalent:

(1) $B_{n}(\Phi, f)$ converges in $C(Y)$ for each $f$ in $C(X)$.

(2) $\Phi$ satisfies condition A.

Proof. We note first that condition A identifies $\Phi$ as a Hausdorff moment sequence from $X$ into $C(Y)[1$, Lemma 8]. (Reference 8 in

Received by the editors April 14, 1966. 
[1] is reference [2] of this paper.)

Suppose (2) holds, i.e., $\Phi$ is a Hausdorff moment sequence from $X$ in to $C(Y)$. By the proof of the first part of [1, Theorem 3], there exists a continuous linear transformation $T$ from $C(X)$ into $C(Y)$ such that, for each $g$ in $C(X), T(g)(s)=\int_{0}^{1} d_{t} K(s, t) \cdot g(t)$ and such that $T(f \cdot x)(s)=\int_{0}^{1} d_{t} K(s, t) \cdot f(t) \cdot x$ for each $f$ in $C(R)$ and each $x$ in $X$, where $R$ denotes the real field and such that, furthermore, $\phi_{m}(s) \cdot x$ $=T\left(t^{m} \cdot x\right)$ for $m=0,1,2, \cdots$ and each $x$ in $X$. We then have that

$$
\begin{aligned}
B_{n}(\Phi, f) & =\sum_{m=0}^{n}\left(\begin{array}{c}
n \\
m
\end{array}\right)\left[\Delta^{n-m} \phi_{m}\right] \cdot f\left(\frac{m}{n}\right) \\
& =T\left[\sum_{m=0}^{n}\left(\begin{array}{c}
n \\
m
\end{array}\right)\left[\Delta^{n-m} t^{m}\right] f\left(\frac{m}{n}\right)\right]=T\left[B_{n}(f)\right]
\end{aligned}
$$

and since $T$ is continuous and $B_{n}(f)$ converges in norm to $f$, we have that $B_{n}(\Phi, f)$ converges in norm to $T(f)=\int_{0}^{1} d K \cdot f$, and hence (1) holds.

Now suppose (1) holds. It is easily seen that for each $n$,

$$
B_{n}(\Phi, f)=\sum_{m=0}^{n}\left(\begin{array}{c}
n \\
m
\end{array}\right)\left[\Delta^{n-m} \phi_{m}\right] \cdot f\left(\frac{m}{n}\right)
$$

defines a continuous linear transformation from $C(X)$ in to $C(Y)$ since it is the finite sum of such transformations. Since $\left\{B_{n}(\Phi, f)\right\}_{n=0}^{\infty}$ converges for each $f$ in $C(X)$, we have by the uniform boundedness principle that there exists a constant $M>0$ such that for each $f$ in $C(X)$ and each $n=0,1,2, \cdots$,

$$
\begin{aligned}
\left\|B_{n}(\Phi, f)\right\|_{C(Y)} & =\left\|\sum_{m=0}^{n}\left(\begin{array}{c}
n \\
m
\end{array}\right)\left[\Delta^{n-m} \phi_{m}\right] \cdot f\left(\frac{m}{n}\right)\right\|_{C(Y)} \\
& \leqq M\left\|_{f}\right\|_{C(X)} .
\end{aligned}
$$

Now suppose a bounded sequence $\left\{x_{m}\right\}_{m=0}^{\infty}$ of points in $X$ is given. There exists a polygonal function $P$ in $C(X)$ which has the values $P(m / n)=x_{m}$ for $m=0,1, \cdots, n$ and $P$ is defined to be linear otherwise. $\|P\|_{C(X)}=\max \left\|x_{m}\right\|$ where the maximum is taken over $m=0, \cdots, n$. Taking $P$ for $f$ above gives

$$
\left\|\sum_{m=0}^{n}\left(\begin{array}{c}
n \\
m
\end{array}\right)\left[\Delta^{n-m} \phi_{m}\right] \cdot x_{m}\right\|_{C(Y)} \leqq M \max _{0 \leqq m \leqq n}\left\|x_{m}\right\| \leqq M \sup _{(m)}\left\|x_{m}\right\|
$$

and (2) holds.

Corollary. For the case in which $Y$ is $X$, (then each $\phi_{m}$ is in $C(B[X, X]))$ the following two statements are equivalent: 
$\left(1^{*}\right) B_{n}(\Phi, f)$ converges to f for each $f$ in $C(X)$.

$\left(2^{*}\right) \phi_{m}(t)=t^{m}$ for $m=0,1, \cdots$.

Proof. Suppose $\left(1^{*}\right)$ holds, then by the theorem, condition $A$ is satisfied and there exists $T$ such that $T\left(t^{m} \cdot x\right)=\phi_{m} \cdot x$ for each $m$, but then by $\left(1^{*}\right) T(f)(s)=\lim _{n \rightarrow \infty} B_{n}(\Phi, f)=f(s)$ for each $f$ in $C(X)$ and hence $T\left(t^{m} \cdot x\right)(s)=s^{m} \cdot x=\phi_{m}(s) \cdot x$ for each $x$ and hence $\phi_{m}(s)=s^{m}$ for all $m$.

The proof that $\left(2^{*}\right)$ implies $\left(1^{*}\right)$ is a trivial modification of the classical proof that $B_{n}(f)$ converges to $f$ for each $f$ in $C(R)$.

\section{BIBLIOGRAPHY}

1. Lynn C. Kurtz and D. H. Tucker, Vector-valued summability methods on a linear normed space, Proc. Amer. Math. Soc. 16 (1965), 419-428.

2. D. H. Tucker, A representation theorem for a continuous linear transformation on a space of continuous functions, Proc. Amer. Math. Soc. 16 (1965), 946-953.

The UNIVERSity OF UTAH 